\title{
CUANDO LOS SABERES NO DIALOGAN. PRÁCTICAS EN CONFLICTO EN EL PARQUE COSTERO SUR (BUENOS AIRES)
}

\author{
When knowledges do not talk. Practices in conflict in the Parque Costero del Sur (Province of \\ Buenos Aires)
}

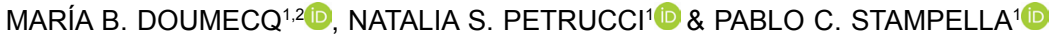

\begin{abstract}
Resumen: El presente trabajo se centró en el partido de Punta Indio (Provincia de Buenos Aires) y tuvo como objetivo relevar las narrativas de los actores involucrados (gestores, docentes y pobladores) sobre el Parque Costero del Sur (PCS) como patrimonio biocultural de la UNESCO. Se aplicaron métodos y técnicas cualitativas usuales en el trabajo etnobiológico. EI PCS es escenario de numerosos conflictos debido a la condición de área protegida habitada. Las principales problemáticas relevadas a través de las narrativas se relacionan con la actividad minera, la pavimentación de la RP № 11, la prohibición del uso de especies nativas, la dicotomía nativo/exótico y las prácticas asociadas, las especies mencionadas y los espacios representativos. Estas problemáticas incluyen tanto aspectos biológicos como culturales. En general, en los pobladores se puede observar cómo ambos aspectos se entrelazan, mientras que en las narrativas de los docentes y gestores del PCS estas dimensiones tienden a separarse. Esta diferencia depende fundamentalmente de la residencia (o no) en la localidad de Punta del Indio, de los intereses económicos y las actividades desarrolladas. Estos intereses impiden el diálogo entre dichos actores, aspecto que puede ser vehiculizado desde la etnobiología.
\end{abstract}

Palabras clave: Comunidad local, conocimiento local, etnobiología, patrimonio biocultural, Punta del Indio.

Summary: This work was carried out in the department of Punta Indio (Province of Buenos Aires) with the aim to collect the narratives of involved actors (managers, teachers and settlers) dealing with the Parque Costero del Sur (PCS) as a UNESCO biocultural heritage. We applied standard qualitative methods and techniques used in ethnobiological research. Numerous conflicts characterize the PCS due to its condition of being a protected area with human settlements. The survey showed that the major problems outlined by the narratives are related to the mining activity, the paving of Provincial Route $\mathrm{N}^{\circ} 11$, the prohibition of exploiting native species, the native / exotic dichotomy and associated practices, which species are mentioned and representative spaces. These problems include both biological and cultural aspects. In general, these two aspects are intermingled in settlers narratives, whereas they tend to be separated in those of teachers and managers of the PCS. This difference depends mainly on residency (or not) in the locality of Punta del Indio, on economic interests and on developed activities. These interests obstruct conversation among the various actors, an aspect that can be facilitated from ethnobiology.

Key words: Biocultural patrimony, ethnobiology, local community, local knowledge, Punta del Indio.

\footnotetext{
${ }^{1}$ Laboratorio de Etnobotánica y Botánica Aplicada, Facultad de Ciencias Naturales y Museo, Universidad Nacional de La Plata, La Plata, Buenos Aires, Argentina. E-mail: mbelendoumecq@gmail.com

${ }^{2}$ Consejo Nacional de Investigaciones Científicas y Técnicas (CONICET). Argentina.
} 


\section{Introducción}

\section{Comunidades locales y áreas protegidas}

A lo largo de la historia, la concepción de áreas protegidas ha cambiado según los marcos conceptuales de distintas corrientes. En principio se basó en la separación intrínseca de la sociedad humana y la naturaleza. Esta concepción ignoraba que muchos de los paisajes que se pretendían preservar eran el resultado de actividades y/o prácticas humanas tradicionales (por ej., roza y quema, tolerancia, protección, agricultura) generadoras de modificaciones en el entorno (Hilgert et al., 2014; Stampella, 2018; Hurrell et al., 2019). En muchos casos, la aplicación de estas ideas obligó a los pobladores locales de zonas protegidas a abandonar tales prácticas tradicionales, siendo incluso algunos de ellos expulsados de su territorio (Trentini, 2015). Algunas de las reacciones ante estas restricciones fueron la sobreexplotación de recursos vegetales y animales (Tuxill et Nabhan, 2001), previamente administradas bajo la estrategia de uso multiple (EUM). La EUM se caracteriza por el manejo de diferentes unidades ecogeográficas (microambientes) y de distintos componentes bióticos y físicos; como también el manejo de procesos ecológicos (Riat et al., 2018).

Hacia la década de 1960, estas ideas conservacionistas comenzaron a ser cuestionadas por investigadores y defensores de los pueblos originarios solicitando se consideraran nuevos métodos para la administración de zonas protegidas. Organizaciones relacionadas con la conservación también establecieron acuerdos que consideraban los derechos de los pobladores locales (Tuxill et Nabhan, 2001). Esto surgió a partir del reconocimiento de los pobladores locales como administradores eficaces de la biodiversidad y del modelado del paisaje a lo largo del tiempo. En este contexto surgieron las Reservas de Biosfera (RB), las cuales plantean, hasta la actualidad, "la compensación y el equilibrio entre mantener la naturaleza y conservar la biodiversidad y la necesidad humana de utilizar los recursos naturales para mejorar el bienestar social y económico de las poblaciones" (Organización de las Naciones Unidas para la Educación, la Ciencia y la Cultura, UNESCO, 2017).
Esta noción de RB enfatiza la importancia de los conocimientos locales y la habilitación al acceso de los recursos por parte de los pobladores locales (Aumeeruddy, 1998; Tuxill et Nabhan, 2001). En líneas generales las RB buscan fomentar la investigación, la observación permanente, la educación y la capacitación, en vinculación con las políticas nacionales y regionales de conservación y desarrollo sostenible. Desde esta perspectiva, las instituciones educativas cumplen un rol fundamental en las distintas actividades desarrolladas por el Comité de Gestión. La gestión en las RB debe ser abierta, dinámica y flexible. En ella se encuentra implícita la concertación de intereses, principalmente por la necesidad de establecer un mecanismo apropiado de participación social para planificar e implementar diversas actividades. Para dar cumplimiento a los objetivos, cada RB posee un plan de manejo. Sus diferentes aspectos se planifican y ponen en funcionamiento por un Comité de Gestión conformado por distintos actores sociales (Paleo et al., 2016). De este modo estos comités son conformados para vehiculizar estos mecanismos y por tal motivo es interesante considerarlos como un ámbito de mediación sobre los conflictos que surjan (Toribio et Soruco de Madrazo, 2005).

La separación entre naturaleza y seres humanos parece haber sido superada con la creación de las $\mathrm{RB}$, ya que la diversidad biológica y la diversidad cultural coevolucionan de manera inseparable (Maffi, 2001). Sin embargo, una nueva dicotomía se fue consolidando en las últimas décadas principalmente desde una mirada conservacionista: las especies nativas versus las especies exóticas. Las primeras como objeto de conservación y las especies exóticas, en cambio, consideradas material disruptivo que debe eliminarse (independientemente de si se conoce la biología y ecología de la especie en cuestión, es decir aspectos de su invasividad). En trabajos etnobotánicos recientes sobre las narrativas de los pobladores locales del noreste, noroeste y centro de Argentina se demostró que las especies introducidas han sido incorporadas como propias y que, a través de procesos locales de selección cultural, han resultado en poblaciones de especies útiles que 
conforman un paisaje particular (Stampella et al., 2013; Hilgert et al., 2014; Lambaré, 2015; Stampella, 2015; Martínez et Manzano-García, 2016; Doumecq, 2019). Por lo tanto, desde la visión local esta dicotomía carece de sentido, mientras que desde la óptica de los gestores de áreas protegidas este punto de vista continúa vigente (Pochettino et al., 2015).

En relación a lo anterior, el presente trabajo tiene como objetivo relevar las narrativas de los actores involucrados (gestores, pobladores locales y docentes) sobre el Parque Costero Sur (PCS) como patrimonio biocultural de la UNESCO, con el fin de aportar al diálogo intersectorial en la gestión de esa RB, escenario de gran diversidad de tareas e intereses como se detalla a continuación.

\section{Área de estudio}

El PCS se encuentra ubicado en el noreste de la provincia de Buenos Aires y ocupa casi todo el sector costero del partido de Punta Indio y un pequeño sector de Magdalena, abarca unas 35.000 ha., en su mayoría de propiedad privada (Fig. 1A). Es una Reserva Mundial de Biosfera Natural y Cultural declarada en 1984 por la UNESCO, a partir de la identificación de un valioso patrimonio biocultural por parte de la fundación CEPA (Centro de Estudios y Proyectación del Ambiente), y de acuerdo con el programa Man and Biosphere (MaB) de la UNESCO (Ghiani Echenique et al., 2018). Dicho programa busca conciliar el desarrollo humano con la valoración del patrimonio natural-cultural a fin de propiciar la conservación de la biodiversidad y el desarrollo sustentable en la producción local.

Uno de los principales motores para la creación del PCS es la presencia del bosque de "tala" (o talar), ecosistema que se extiende a lo largo del litoral en el noreste de la provincia de Buenos Aires y que se halla muy bien representado en este sector sobre los cordones de conchilla (Parodi, 1940; Torres Robles,
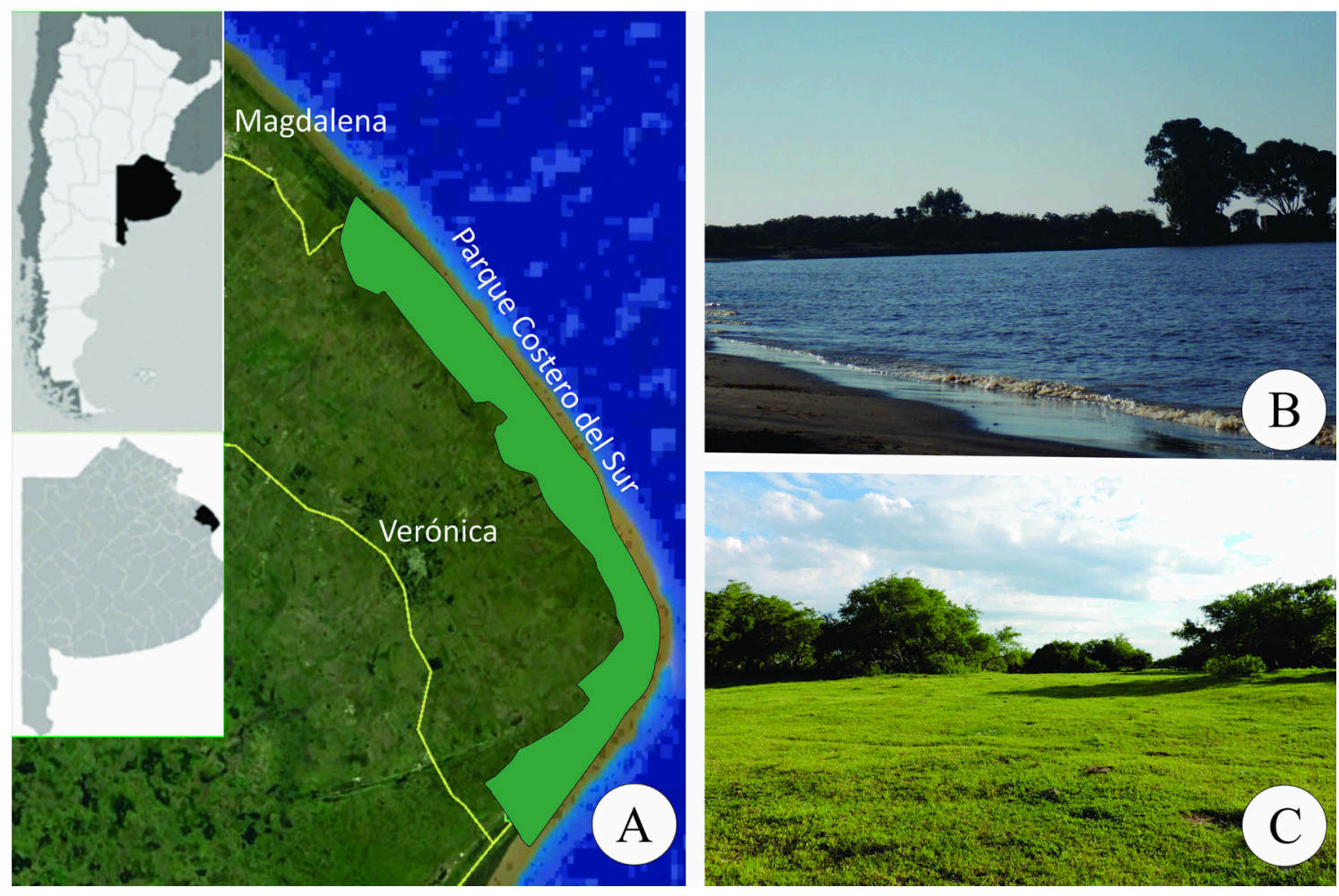

Fig. 1. A: Mapa mostrando la ubicación del PCS. B: Playa. C: Talar.

Fig. 1. A: Map showing location of PCS. B: Beach. C: "Tala" wood formation. 
2009). En la asociación florística de este ecosistema la especie arbórea más conspicua y continua es el "tala" (Celtis tala Gillies ex Planch.). La formación del ambiente comienza en el Holoceno medio (ca. 6000 años AP) cuando el mar desciende progresivamente, determinando la conformación de la llanura costera del noroeste bonaerense. El último descenso relativo del nivel mar se registra en el Holoceno tardío (ca. 3000 años AP) y, en concordancia con otros depósitos marinos de la región, se propuso que a partir de este evento comienzan condiciones de estabilidad y procesos de formación de suelo sobre la región (Paleo et al., 2002). En estos nuevos cordones de conchillas, de aproximadamente 2 metros de altura, localizados entre 1-2 km de la costa del río, se registraron 12 sitios arqueológicos de los cuales ocho han sido estudiados. Las dataciones del área cuentan con una ocupación de ca. 1800 años AP (Pérez Meroni et Paleo, 1999; Paleo et Pérez Meroni, 2004, 2007, 2010). Según Paleo et al. (2016) el talar habría constituido un lugar clave en la elección para el asentamiento por brindar reparo, protección y múltiples recursos.

Según el Censo Nacional de Población, Hogares y Vivienda 2010, el partido de Punta Indio cuenta con 9888 pobladores, siendo la cabecera la localidad de Verónica donde se concentran la mayoría de los habitantes, mientras que el resto de la población se encuentra dispersa. El mayor centro poblacional dentro del PCS lo constituye la localidad de Punta del Indio, que cuenta con aproximadamente 569 pobladores, aunque algunas personas no residen de forma permanente (INDEC, 2010). En los últimos años se ha registrado la llegada de habitantes provenientes principalmente del gran Buenos Aires con nuevos hábitos y costumbres. Las principales actividades económicas son la ganadería, la extracción de conchilla en los campos privados $\mathrm{y}$, actualmente, el turismo (Ghiani Echenique et al., 2018) (Fig. 2).

Por tal motivo, el PCS se constituye como una reserva de protección parcial categoría VI Unión Internacional para la Conservación de la Naturaleza (UICN), es decir que permite el aprovechamiento sostenible de los ambientes (Stampella et al., 2016). A nivel provincial fue declarado Refugio de Vida Silvestre (año 1997), debido a su riqueza en biodiversidad, donde la presencia del bosque costero de talares se conjuga con bañados y playas (Fig. 1B y C). A nivel local, existen ordenanzas municipales de Punta Indio que prohíben la utilización de ciertas especies arbóreas nativas típicas del talar como "tala", "molle" [Schinus longifolius (Lindl.) Speg.], "coronillo" (Scutia buxifolia Reissek), "sombra de toro" [Jodina rhombifolia (Hook. \& Arn.) Reissek], "espinillo" [Vachellia caven (Molina) Seigler \& Ebinger] y "ombú" (Phytolacca dioica L.) (Ordenanza $\mathrm{N}^{\circ}$ 294/98 Punta Indio). Esta normativa suele ser cuestionada por los pobladores locales que reclaman el derecho de seguir utilizando algunas de estas especies nativas como "tala" y "coronillo", muy valoradas como leña (Doumecq, 2019). Por otro lado, la municipalidad de Magdalena, promueve la conservación a través de beneficios tributarios para quienes preservan la flora nativa en su propiedad (Ordenanza No 761/2914). Existen diferentes organizaciones no gubernamentales (ONG) en la localidad de Punta del Indio como por ejemplo la Asociación Civil "Amigos del Parque Costero del Sur", la cual se conformó con el objetivo de trabajar en la puesta en valor del PCS mediante la ejecución de diferentes proyectos. Conjuntamente con los municipios y con la participación de los pobladores se está trabajando en la reproducción de plantas autóctonas para la reforestación, realización de cartelería, construcción de senderos y de un centro de interpretación ambiental. Asimismo, algunos pobladores locales independientes realizan talleres y caminatas para el reconocimiento de plantas silvestres comestibles, jornadas de control de exóticas y de valoración de la flora y fauna nativa (Ghiani Echenique et al., 2018).

Dado que el PCS, como se mencionó anteriormente, está conformado mayormente por propiedades privadas, su conservación depende de las decisiones que tomen sus propietarios. Es de destacar, que la presencia del talar no obstaculiza la ganadería, por el contrario, brinda alimento y reparo a los animales, y se considera que esta actividad hizo posible la permanencia de los talares hasta nuestros días (Arturi et al., 2006). Esta 

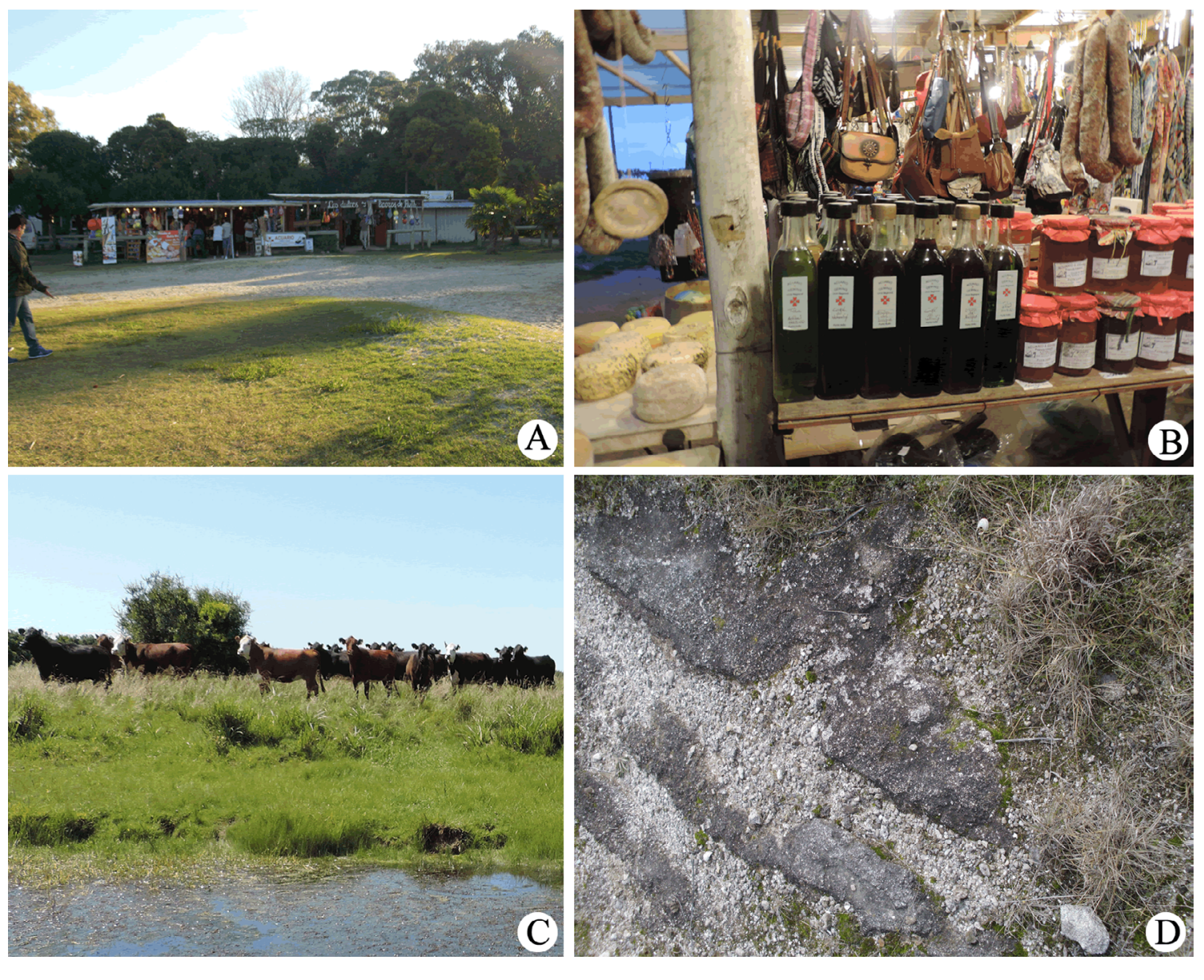

Fig. 2. Principales actividades económicas. A: Venta de artesanías y productos en puestos de la playa. B: Productos elaborados con tala. C: Actividad ganadera. D: Extracción de conchilla.

Fig. 2. Main economic activities. A: Sale of crafts and products in stalls on the beach. B: Products made from tala tree wood. C: Livestock activity. D: Seashell extraction.

tendencia sufrió algunos cambios a partir de la década de 1990. Por razones económicas, los campos se subdividieron, hubo recambio de propietarios y, muchos de ellos, se volcaron a la actividad minera. Cabe destacar que para la extracción de conchilla es necesario derribar el talar que crece sobre este sustrato, por lo que la presión sobre esta formación vegetal se fue incrementando en los últimos años (Paleo et al., 2016). Además, con la extracción de conchilla se eliminan los reservorios de agua dulce que se encuentran en forma de lentes en los cordones conchiles, afectando la sustentabilidad hídrica del área (Tejada et al., 2011).

Hoy en día, el parque se encuentra en el proceso de renovación del título de RB. Luego de varios intentos, en noviembre de 2014, se conformó un Comité de Gestión que solicitó en forma explícita el asesoramiento de diferentes grupos de investigación de la Universidad Nacional de La Plata (Paleo et al., 2016). Además, se convocó a la Fundación CEPA para que reúna la documentación necesaria para la elaboración de un plan de manejo que permita obtener la renovación del Título de RB. Este plan se presentó en diciembre de 2018 y actualmente se encuentran a la espera de una respuesta por parte de la UNESCO. Este proceso no ha sido sencillo, por un lado por la dificultad que implica el manejo bipartito (entre Magdalena y Punta Indio) del PCS y por otro por la diversidad de actores e intereses involucrados. 


\section{Materiales y Métodos}

El trabajo de campo se llevó a cabo durante los años 2017 y 2018. Previo consentimiento informado, se realizaron 20 entrevistas semiestructuradas a pobladores locales, docentes y gestores municipales, de ambos sexos, entre 30 y 65 años de edad. Se aplicaron métodos y técnicas cualitativas usuales en el trabajo etnobiológico: entrevistas semiestructuradas, listados libres de especies "a conservar" y "a eliminar", y caminatas con los entrevistados (Albuquerque et al., 2019). La selección de los mismos se realizó a través de la técnica "bola de nieve" (Bernard, 2000). La información relevada durante las entrevistas fue registrada en libretas de campo y medios audiovisuales (grabador y cámara fotográfica).

También se realizaron talleres en el ámbito educativo (nivel preescolar, primario $\mathrm{y}$ secundario) en el marco del proyecto de extensión "Todas las voces del parque. Construcción participativa del patrimonio natural-cultural del Ecocentro del Parque Costero del Sur (Partido de Punta Indio, Buenos Aires)" desarrollado durante el año 2017. Dicho proyecto, surgió a partir de la demanda del Municipio de Punta Indio y tuvo como objetivo generar espacios participativos para promover la valorización del patrimonio natural-cultural con el fin de plasmar múltiples voces en la generación de un guión museográfico $\mathrm{y}$ adecuación del Sendero de Interpretación Municipal. En estas actividades participaron el Jardín de infantes $\mathrm{N}^{\circ}$ 904, la Escuela Primaria $\mathrm{N}^{\mathrm{o}} 8$ y la Escuela Secundaria Básica $\mathrm{N}^{\mathrm{o}} 3$ de la localidad de Punta del Indio, y la Escuela Primaria $\mathrm{N}^{\mathrm{o}} 14$ del paraje Punta Piedras. Algunos de los estudiantes de las escuelas pertenecen al grupo de pobladores llegados en los últimos años al parque. Con los estudiantes de primaria se trabajó con mapas del partido de Punta Indio con el objetivo de localizar espacios identitarios para ellos, también se elaboraron personajes e historias de Punta Indio a través de la confección títeres e historietas. Por otra parte, con los estudiantes de secundaria, se realizaron actividades donde se relevaron propuestas concretas de mejoras del Sendero de Interpretación Municipal. También se asistió al taller participativo organizado por la fundación CEPA para la renovación del título de RB (Fig. 3).
En el laboratorio se procedió a transcribir y analizar las entrevistas realizadas. También se analizaron los resultados obtenidos en los talleres. Se identificaron lugares considerados identitarios así como las especies de animales y vegetales mencionados. Para el tratamiento nomenclatural actualizado de las especies vegetales identificadas se consultaron las bases de datos del IBODA (2018). La información relevada fue organizada en diferentes bases de datos empleando los programas Microsoft Office Excel 2007.

\section{Resultados y Discusión}

Los resultados obtenidos en las entrevistas y talleres se presentan de manera comparativa y sintética en la Tabla 1 y se discuten a continuación.

En general, se relevaron acuerdos entre los pobladores y los gestores en lo relacionado a la práctica extensiva de agricultura y ganadería, y la promoción de las pequeñas producciones intensivas. Asimismo, pobladores y docentes acordaron en lo relativo a la contaminación del agua, la sobrepesca, la urbanización, la caza y en la puesta en valor de espacios comunes. Existe un acuerdo general entre las tres categorías de actores en cuanto a la preocupación por la erosión de costa, difiriendo en la forma de abordar la solución si bien la mayoría concuerda con la implantación de juncales (Tabla 1).

En relación a los desencuentros, se pueden apreciar discursos -o bien aspectos de los mismos- contrapuestos en lo relativo a los aspectos tratados a continuación:

1) Actividad minera (conchilla). Posiblemente este aspecto sea uno de los más conflictivos ya que desde la perspectiva de la gestión del PCS e investigaciones se trata de una de las actividades más perniciosas para la conservación y uso sustentable de los talares (Arturi et al., 2006; Stampella et al., 2016; Ghiani Echenique et al., 2018). Si bien las ordenanzas municipales no contemplan estas problemáticas, utilizándose este sedimento para la construcción de calles y caminos, inclusive la RP $N^{o} 11$ que atraviesa el PCS, las autoridades actuales tratan de atender la situación mencionada evitando su utilización. Esto generó un desacuerdo particular en lo que hace al acondicionamiento del Sendero de Interpretación, dado que los estudiantes 

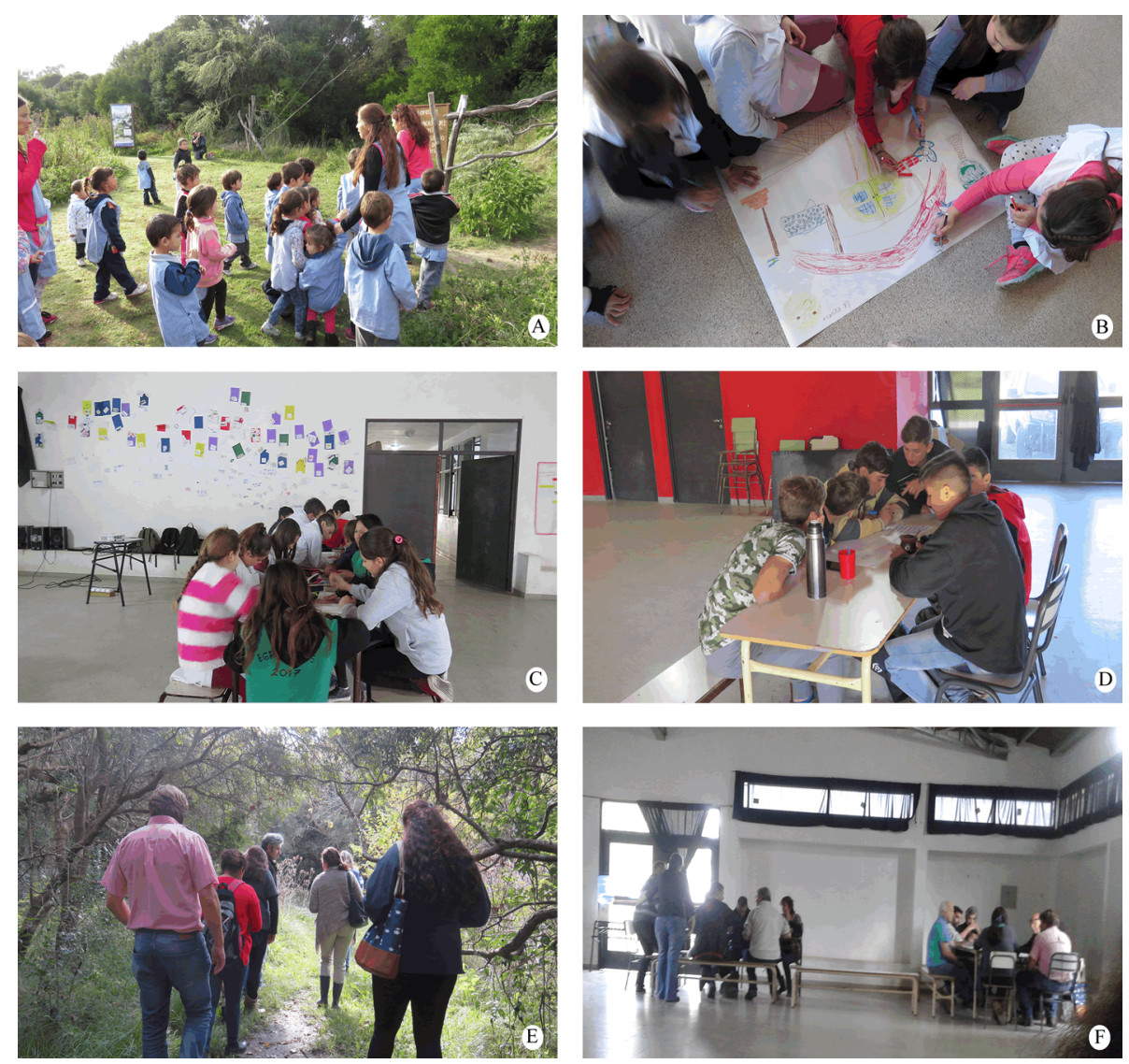

Fig. 3. A: Caminata por el Sendero de Interpretación Municipal con los niños y maestros de Jardín de Infantes $\mathrm{N}^{\circ} 904$. B-C: Talleres con los estudiantes de la Escuela Primaria No 8 y No 14. D: Talleres con los estudiantes de la Escuela Secundaria Básica $\mathrm{N}^{\circ}$ 3. E: Caminata por el Sendero de Interpretación Municipal con gestores municipales del parque. F: Taller abierto a toda la comunidad organizado por la fundación CEPA para la renovación del título de reserva de Biósfera.

Fig. 3. A: Walks through the Municipal Interpretation Path with children and teachers of the Kindergarten No 904 . B-C: Workshops with students of Primary School No 8 and $N^{o} 14$. D: Workshops with students of Basic Secondary School $\mathrm{N}^{\circ}$ 3. E: Walks through the Municipal Interpretation Path with municipal administration agents. F: Workshop open to the entire community organized by the CEPA foundation for the renewal of the Biosphere Reserve title.

propusieron elevar el sendero agregando conchilla para mejorar su transitabilidad y desde el Municipio desestimaron la propuesta por las razones expuestas.

2) Pavimentación de la $R P N^{o}$ 11. En líneas generales el disenso en torno a la pavimentación de la ruta de acceso al PCS se vincula con la aceptación o rechazo del incremento del turismo que se vería facilitado por un mejor camino. Los argumentos a favor son la mayor actividad económica que representaría el turismo, aunque también plantean una mejor calidad en la asistencia médica para los pobladores locales. Los opositores en cambio sostienen que la llegada de muchos turistas será perjudicial para la preservación del PCS, ya que generaría más basura y mayor tránsito (y con ello una mayor probabilidad de accidentes hacia los residentes y fauna local). Este último argumento coincide con lo estudiado por Salleras (2013) en la Quebrada de Humahuaca (Jujuy, noroeste de Argentina) donde el incremento de una actividad turística que ignora el patrimonio biocultural provoca impactos que hacen dudar la sostenibilidad de la actividad a largo plazo. 
Tabla 1. Síntesis y comparación de los ejes abordados según los distintos actores.

Table 1. Synthesis and comparison of the axes addressed between the different social actors.

\begin{tabular}{|c|c|c|c|}
\hline Ejes abordados & Gestores & Pobladores & Docentes \\
\hline \multicolumn{4}{|c|}{ Conservación de flora y fauna } \\
\hline Actividad minera & $\begin{array}{l}\text { Consenso entre gestores } \\
\text { en que no abran nuevas } \\
\text { canteras. Proponen } \\
\text { mayor control sobre estas } \\
\text { actividades y compensación } \\
\text { económica para que } \\
\text { conserven el bosque nativo. }\end{array}$ & $\begin{array}{l}\text { Disenso entre los } \\
\text { pobladores en la } \\
\text { extracción y utilización de } \\
\text { conchilla. }\end{array}$ & $\begin{array}{l}\text { Solo un docente } \\
\text { mencionó esta actividad } \\
\text { pero no sabía que } \\
\text { había canteras dentro } \\
\text { del parque. }\end{array}$ \\
\hline $\begin{array}{l}\text { Agricultura y } \\
\text { ganadería }\end{array}$ & $\begin{array}{l}\text { Proponen mantener las } \\
\text { actividades de manera } \\
\text { extensiva y la compensación } \\
\text { económica. }\end{array}$ & $\begin{array}{l}\text { Consenso entre los } \\
\text { pobladores en la } \\
\text { realización de estas } \\
\text { actividades de manera } \\
\text { extensiva. }\end{array}$ & NS/NC. \\
\hline $\begin{array}{l}\text { Pequeñas } \\
\text { producciones } \\
\text { intensivas }\end{array}$ & $\begin{array}{l}\text { Promoción de la apicultura, } \\
\text { avicultura en chacras, huertas } \\
\text { orgánicas, turismo rural con } \\
\text { control de caza y pesca con } \\
\text { red. }\end{array}$ & $\begin{array}{l}\text { Muchos pobladores } \\
\text { viven del turismo rural } \\
\text { por lo que acuerdan con } \\
\text { la promoción de estas } \\
\text { actividades. }\end{array}$ & NS/NC. \\
\hline $\begin{array}{l}\text { Sobrepesca, caza, } \\
\text { pavimentación } \\
\text { y aumento de la } \\
\text { urbanización }\end{array}$ & $\begin{array}{l}\text { Proponen mayor difusión } \\
\text { y presencia de agentes } \\
\text { ambientales municipales. } \\
\text { Aplicación de la legislación } \\
\text { vigente. Evitar la } \\
\text { construcción del murallón y la } \\
\text { pavimentación de la RP } N^{\circ} 11 .\end{array}$ & $\begin{array}{l}\text { Disenso entre los } \\
\text { pobladores en torno a la } \\
\text { pavimentación de la RP } \\
\mathrm{N}^{\circ} 11 \text { y a la pesca con red } \\
\text { y trasmallo. }\end{array}$ & $\begin{array}{l}\text { Disenso entre los } \\
\text { docentes en la } \\
\text { pavimentación de la RP } \\
\text { № 11. Falta de control } \\
\text { sobre la caza. Falta } \\
\text { de participación de los } \\
\text { pobladores. }\end{array}$ \\
\hline Erosión de la costa & $\begin{array}{l}\text { Proponen evitar pérdida } \\
\text { del junco (Schoenoplectus } \\
\text { californicus (C. A. Mey.) } \\
\text { Soják) debido al pastoreo. } \\
\text { Colocación de rocas sobre la } \\
\text { costa para evitar la erosión. }\end{array}$ & $\begin{array}{l}\text { Distintas opiniones entre } \\
\text { productores ganaderos y } \\
\text { el resto de la población. } \\
\text { Grupos autoconvocados } \\
\text { juntan firmas para poder } \\
\text { plantar juncos en la playa. }\end{array}$ & $\begin{array}{l}\text { Proyecto impulsado } \\
\text { por vecinos para } \\
\text { replantar juncos en } \\
\text { la playa con el fin de } \\
\text { evitar que el río siga } \\
\text { avanzando. No están a } \\
\text { favor de la colocación } \\
\text { de rocas en la costa } \\
\text { ya que las mismas han } \\
\text { ocasionado accidentes. }\end{array}$ \\
\hline $\begin{array}{l}\text { Especies } \\
\text { mencionadas y } \\
\text { valoraciones }\end{array}$ & $\begin{array}{l}\text { Dicotomía entre especies } \\
\text { exóticas y nativas. Especies } \\
\text { exóticas invasoras como } \\
\text { "laurel", "mora" y "jabali". } \\
\text { "Ligustro" debe ser } \\
\text { talado. "Mariposa bandera } \\
\text { argentina"= "coronillo" } \\
\text { (símbolo del parque). }\end{array}$ & $\begin{array}{l}\text { "Tala" y } \\
\text { "coronillo"=leña=asado. } \\
\text { "Pastos", "juncos", } \\
\text { "ligustro","peces", } \\
\text { "caballo", } \\
\text { "chancho","jabalí" (Sus } \\
\text { scrofa), "gato montés", } \\
\text { "lagarto" (Salvator } \\
\text { merianae), "colibri", } \\
\text { "perro", "tuco-tuco del } \\
\text { talar" (Ctenomys talarum). }\end{array}$ & $\begin{array}{l}\text { Plantas y animales } \\
\text { nativos ("mariposa } \\
\text { bandera argentina"= } \\
\text { "coronillo" = símbolo } \\
\text { del parque; "tala" y } \\
\text { "ciervo"). Acuerdan } \\
\text { en la prohibición } \\
\text { de uso de especies } \\
\text { nativas, excepto en la } \\
\text { extracción de árboles } \\
\text { para construcción de } \\
\text { viviendas. }\end{array}$ \\
\hline
\end{tabular}




\begin{tabular}{|c|c|c|c|}
\hline Ejes abordados & Gestores & Pobladores & Docentes \\
\hline Especies exóticas & $\begin{array}{l}\text { Algunos proponen la } \\
\text { realización de jornadas de } \\
\text { extracción de las mismas y } \\
\text { aprovechamiento productivo } \\
\text { de especies madereras y } \\
\text { proponen principal atención a } \\
\text { especies invasoras. }\end{array}$ & $\begin{array}{l}\text { Disenso entre los } \\
\text { pobladores en la } \\
\text { utilización y extracción } \\
\text { de especies exóticas. } \\
\text { Conflicto por el uso } \\
\text { de glifosato. Algunos } \\
\text { pobladores reclaman el } \\
\text { uso de las nativas. }\end{array}$ & NS/NC. \\
\hline \multicolumn{4}{|c|}{ Puesta en valor de espacios comunes } \\
\hline Lugares identitarios & $\begin{array}{l}\text { Sendero de interpretación } \\
\text { Municipal, Parque Costero } \\
\text { del Sur, Sendero Villoldo, } \\
\text { campos (turismo rural), playa, } \\
\text { Sociedad de Fomento, Ruinas } \\
\text { del hotel. }\end{array}$ & $\begin{array}{l}\text { Sendero Villoldo, } \\
\text { mirador, mangrullo, } \\
\text { sangrado, playa Sarandí } \\
\text { y Picaflor, campo, hotel } \\
\text { abandonado, estancias, } \\
\text { bosque, delegación, } \\
\text { sociedad de fomento, } \\
\text { parrillas, paisaje=río. }\end{array}$ & $\begin{array}{l}\text { Monte de tala, playa } \\
\text { "El Pericón", "Sarandí", } \\
\text { Sendero Villoldo, } \\
\text { Sendero Municipal, } \\
\text { calles del pueblo y } \\
\text { ruinas del hotel. }\end{array}$ \\
\hline $\begin{array}{l}\text { Acondicionamiento } \\
\text { del Sendero de } \\
\text { Interpretación } \\
\text { Municipal }\end{array}$ & $\begin{array}{l}\text { Acuerdan en no colocar } \\
\text { elementos recreativos ni } \\
\text { cestos de basura. Colocar } \\
\text { cartelería informativa sobre } \\
\text { especies nativas. }\end{array}$ & $\begin{array}{l}\text { Acuerdan en colocar } \\
\text { cestos de basura, juegos, } \\
\text { mesas y cartelería } \\
\text { informativa sobre flora y } \\
\text { fauna. }\end{array}$ & $\begin{array}{l}\text { Acuerdan en colocar } \\
\text { cestos de basura con } \\
\text { separación de residuos, } \\
\text { mesas y cartelería } \\
\text { informativa sobre } \\
\text { especies nativas. }\end{array}$ \\
\hline
\end{tabular}

Referencias. NC: No contesta, NS: No sabe.

3) Prohibición del uso de especies nativas. La ordenanza N N $^{0}$ 294/98 de Punta Indio prohíbe la utilización de las especies nativas del talar. Muchas de estas especies nativas prohibidas son localmente valoradas como leña, y se genera una gran contradicción a escala individual con respecto al cumplimiento de la ordenanza: mientras que, muchos pobladores del PCS evitan consumir esta leña para preservar las especies protegidas, otros pobladores argumentan que tienen derecho a utilizarlas ya que viven en la zona desde antes de la existencia del PCS (Doumecq, 2019).

Por ello, la ordenanza mencionada resulta contradictoria con la definición de las RB, ya que dificulta el desarrollo sustentable de la comunidad local. Diversos estudios realizados en áreas protegidas han demostrado que la prohibición en el uso de las especies nativas conduce a la interrupción de las relaciones entre los pobladores locales y el entorno. Esta interrupción, a la vez, provoca el empobrecimiento de las familias, la pérdida de procesos culturales de selección y criterios de manejo generadores de biodiversidad, como también la diversidad misma de especies, variedades y cultivares (Aumeeruddy, 1998; Tuxill et Nabhan, 2001; Stampella, 2018).

4) Dicotomía nativo/exótico y las prácticas asociadas (inducción/extracción). Diferentes animales como "chancho" y "gato montés" (Leopardus geoffroyi), y plantas de los alrededores como "ligustro" (Ligustrum lucidum W. T. Aiton) y "tala" son mencionados por los pobladores como propios de su entorno, independientemente de su origen. Entre ellos, las especies exóticas -primeramente utilizadas y varias de ellas naturalizadas- son resignificadas y consideradas como parte del paisaje y no como elementos disruptivos, tal como plantean Hilgert et al. (2014), para los frutales introducidos del noreste y noroeste argentino. Los gestores distinguen entre ambas categorías y todas las acciones de conservación giran en torno a las especies nativas y el ecosistema que las alberga, tal como ha sido mencionado por Pochettino et al. (2015). Además, en el discurso de los gestores, se distingue dentro de las especies exóticas aquellas que son invasoras y que representan, según su visión, una 
amenaza para la conservación del PCS. También existen algunos pobladores conservacionistas -correspondiente al grupo de nuevos pobladores del parque- que distinguen entre ambas categorías. Los gestores y estos pobladores organizan diversas actividades para restaurar los talares (jornadas de extracción de especies exóticas y aprovechamiento de las mismas). Estas acciones en algunos casos resultaron conflictivas por una parte, por el método empleado, principalmente el uso de herbicida (glifosato) para eliminarlas y, por otra parte, debido a que muchos pobladores se oponían a la extracción de estas especies. La continuidad de estas medidas ha resultado en espacios que localmente se denominan "jardines silvestres", es decir los jardines particulares de algunos de estos nuevos pobladores donde se promueven las especies nativas y se controlan las exóticas. Esto coincide con lo planteado por Martínez et Manzano-García (2016) para los pobladores foráneos de las sierras de Córdoba, quienes tenían más presente esta dicotomía, y propiciaban el avance y reforestación con la flora nativa, controlando y eliminando la flora exótica invasora.

Sin embargo, desde hace relativamente poco tiempo, se observa un cambio en el discurso de algunos gestores en relación a las especies exóticas con respecto a trabajos previos en el área (Pochettino et al., 2015; Stampella et al., 2016; Ghiani Echenique et al., 2018). Como ejemplo, uno de los gestores mencionaba: "Estamos tratando de convencernos que hay muchas cosas que ya son parte del parque, por eso esto de nativo/exótico creemos que es necesario dejarlo un poco al costado" (G. D. 51 años, masculino, 2018).

5) Especies mencionadas y sus valoraciones. Los gestores y docentes destacan el "coronillo" y a la mariposa "bandera argentina" (Morpho epistrophus argentinus) como símbolos del parque, mientras que para los pobladores, en general, son más importantes tanto el "coronillo" como el "tala", valorados por su utilidad como leña en la calefacción de los hogares y cocción de alimentos (Doumecq, 2019). De esta manera, podemos decir que entre los distintos actores cambian las representaciones de los elementos identitarios del paisaje local, entendido este último como el espacio interpretado por los pobladores (Ingold, 1993).
En relación a este tópico, las instituciones educativas cumplieron un rol como articuladoras de saberes entre los gestores y pobladores, donde la dinámica fue unidireccional. En estos ámbitos el reconocimiento de los saberes locales, en este caso representado por los estudiantes, muchas veces es una cuestión problemática debido a que se emplean marcos pedagógicos cercanos a la educación denominada bancaria, es decir imposición de conocimientos decididos en otros ámbitos en lugar de conocimientos construidos con los estudiantes y en función de los saberes previos como corresponde al modelo de la educación liberadora sostenido por Paulo Freire (Freire, 2005; Riat, 2015). Este tipo de trabajos revaloriza fundamentalmente el contexto local y los educadores se posicionan como parte del equipo que planifica y coordina las distintas actividades, relacionadas a la vez con los contenidos curriculares (Riat, 2015). En el área de estudio, en cambio, los docentes de nivel secundario entrevistados no tenían conocimiento de la existencia del Comité de Gestión del PCS ni de las actividades que promueven dado que nunca han sido convocados a las reuniones realizadas. Sólo tienen conocimiento de algunas actividades de conservación y prevención realizadas por pobladores o por algunas $\mathrm{ONG}$ locales (Amigos del PCS y COMCOSUR). A modo de superación de esta situación, los docentes entrevistados plantearon la necesidad del dictado de charlas por parte del Comité de Gestión dando cuenta del avance de los temas relacionados a la reserva, en forma sistemática y no voluntarista.

6) Espacios representativos y Sendero de Interpretación Municipal. Aquellos lugares mencionados por los pobladores en general son espacios transitados, visitados, ocupados, intervenidos, vividos, es decir, identificados por su cotidianeidad; mientras que para los gestores son aquellos espacios a conservar y/o restaurar, entendidos como espacios prístinos (en el sentido de la vuelta al pasado, a "lo natural"). De esta manera, como plantean Greider et Garkovich (1994) estos lugares representativos para los pobladores constituyen espacios simbólicos producto de la interacción entre estos y el entorno de acuerdo a distintas creencias y valoraciones. El predio elegido por el Municipio para la construcción del Sendero 
de Interpretación (Fig. 4), no era conocido para la mayoría de los pobladores entrevistados o bien conocían su existencia, pero nunca lo habían visitado. Sin embargo, en los talleres realizados en las escuelas se propusieron una serie de elementos (mesas, bancos, cestos de
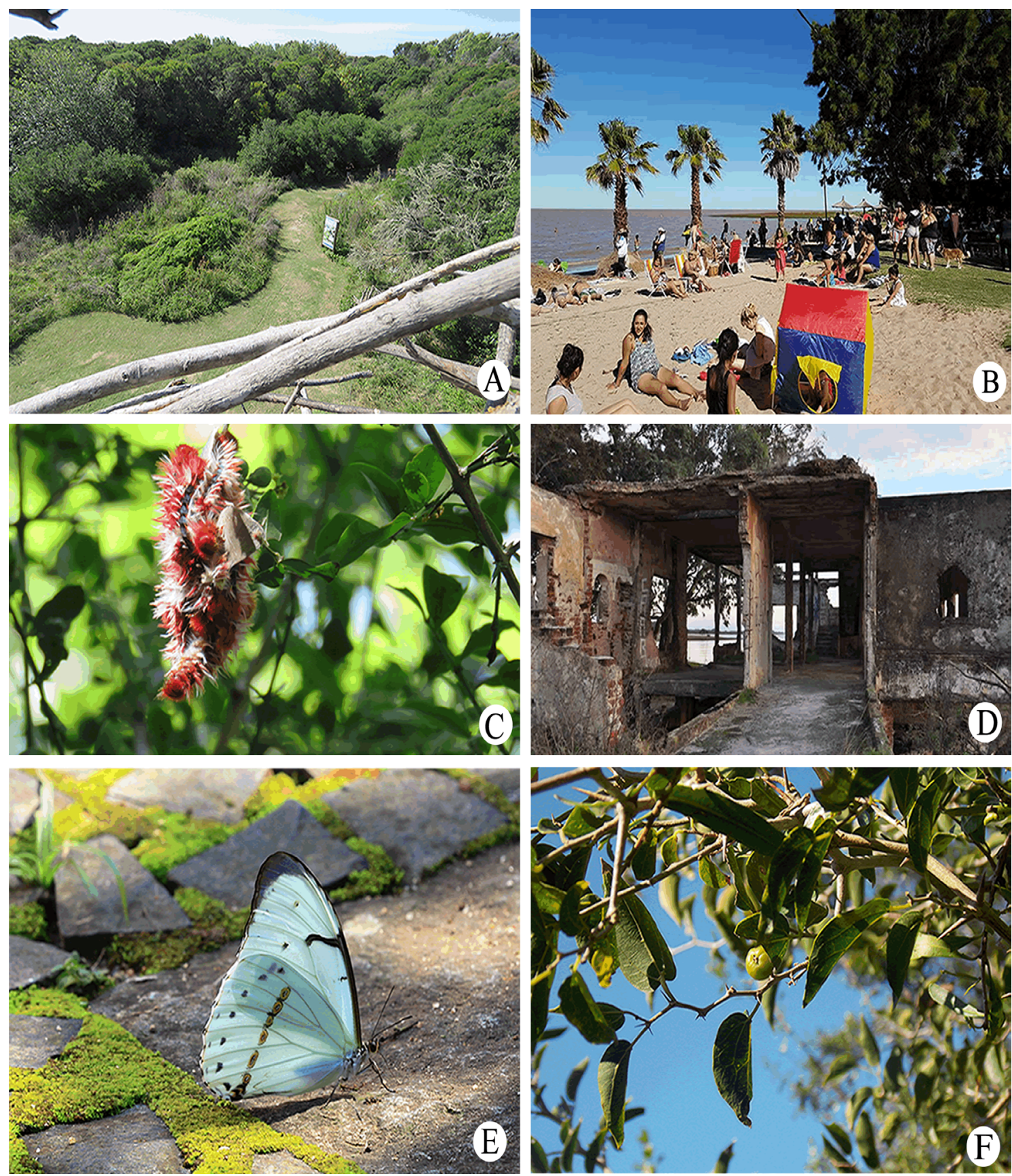

Fig. 4. Lugares y especies identitarios mencionado por los pobladores. A: Sendero Villoldo. B: Playa El Pericón. C: Oruga de mariposa "bandera argentina". D: Ruinas del Hotel Argentino. E: Mariposa "bandera argentina". F: "Tala". Fig. 4. Identitary sites and species mentioned by local inhabitants. A: Villoldo Path. B: Pericón Beach. C: "Argentine flag" butterfly (caterpillar). D: Ruins of the Argentino Hotel. E: "Argentine flag" butterfly (adult). F: "Tala" tree. 
basura, luces, parrillas, entre otros) que podrían posibilitar la apropiación de dicho espacio, otorgándoles identidad a este sendero (Ghiani Echenique et al., 2018). Desde la gestión argumentaron que esto no era posible ya que la función del sendero era sólo para tránsito. Nuevamente estas diferencias marcan los distintos significados de identidad. Los gestores eligen un espacio que represente al parque incorporando elementos con cierta información (principalmente especies nativas), sin tener en cuenta la participación de los pobladores.

Las situaciones descriptas refieren a desencuentros comunes entre los distintos actores que habitan el parque. Tales disputas radican principalmente en la condición de área protegida habitada, la cual confronta la conservación con las actividades económicas familiares y empresariales, tal como fue planteado por Stampella et al. (2016), en un trabajo realizado en la zona de estudio sobre la valoración local del cambio ambiental.

\section{Conclusiones}

De lo expuesto se deriva que existen diferentes narrativas entre y dentro de los distintos grupos de actores que componen la comunidad del PCS, las cuales incluyen tanto aspectos biológicos como culturales. En los pobladores locales se puede observar cómo ambos aspectos se entrelazan, mientras que en general la visión de los docentes y gestores estas dimensiones tienden a separarse.

Estas diferencias en las narrativas podrían deberse al lugar de residencia de los distintos actores, por lo general, los docentes y gestores no habitan en Punta del Indio. Esto también se observa en las discrepancias entre los pobladores, donde el discurso de los nuevos habitantes se aproxima más al de los gestores. La permanencia en el lugar genera una relación diacrónica con el ambiente, resultando a lo largo del tiempo en la construcción del paisaje local. Otro factor que incide en las narrativas son los intereses económicos y las actividades desarrolladas. Estos intereses impiden el diálogo fluido y constructivo entre dichos actores, diálogo que sin embargo logra restablecerse frente a problemas comunes tales como la erosión costera.
A lo largo de este trabajo pudimos apreciar cambios en el discurso sobre las especies exóticas por parte de los gestores del PCS. Esta perspectiva -ya reconocida en los pobladores locales- constituye un insumo de relevancia en la gestión de políticas que tiendan a romper la drástica separación entre sociedad y naturaleza.

Como contribución, entendemos que la etnobiología, en tanto construye conocimiento científico sobre los saberes ambientales situados, de manera interdisciplinaria, constituye una eficaz herramienta a partir de la cual entender el conocimiento y manejo de los recursos. Así, pone en diálogo la perspectiva académica con las voces locales, al mismo tiempo que las hace disponibles para la población en general. Por ello, los etnobiólogos tienen un compromiso indelegable con las comunidades con las que interactúan, entendiendo en este caso particular nuestro trabajo como una instancia facilitadora de la comunicación y del accionar conjunto de todos los habitantes del PCS.

\section{Agradecimientos}

A los pobladores que participaron en las entrevistas. A la Dra. M. Lelia Pochettino por la lectura crítica del manuscrito y sus acertados comentarios. A todos los miembros del Proyecto de Extensión "Todas las voces del Parque" y a la Universidad Nacional de La Plata quien financió y avaló dicho proyecto (Expediente Código 100. No 13.306. Año 2016).

\section{Bibliografía}

ALBUQUerQUe, U., R. PAIVA DE LUCENA, L. FERNANDES DA CUNHA \& R. NÓBREGA (eds.). (2019). Methods and techniques in Ethnobiology and Ethnoecology. 2nd edition. Humana Press, Springer, New York. 342 pp.

https://doi.org/10.1007/978-1-4939-8919-5

ARTURI, M. F., C. A. PÉREZ, M. HORLENT, J. F. GOYA \& S. S. TORRES ROBLES. (2006). El manejo de los talares de Magdalena y Punta Indio como estrategia para su conservación. En MÉRIDA, E. \& J. ATHOR (eds.), Talares Bonaerenses y su Conservación, pp. 37-45. Fundación de Historia Natural Félix de Azara, Buenos Aires. 
AUMEERUDDY, Y. (1998). Modos rurales de representación y gestión de los sistemas agrosilvícolas en la periferia del Parque Nacional Kerinci Seblat, Sumatra, Indonesia. Documento de Trabajo de Pueblos y Plantas No 3. París, UNESCO. 46 pp.

BERNARD, R. (2000). Social research methods. Qualitative \& quantitative approaches. California, Sage, Thousand Oaks. 93 pp.

DOUMECQ, M. B. (2019). Evaluación etnobotánica de los recursos vegetales combustibles actuales y potenciales de la Ribera Platense (Provincia de Buenos Aires, Argentina). Tesis Doctoral, Universidad Nacional de La Plata, Argentina. 209 pp.

FREIRE, P. (2005). Pedagogía del oprimido. 2a ed., Siglo XXI Editores, Buenos Aires.

GHIANI ECHENIQUE, N., M. B. DOUMECQ \& M. L. POCHETTINO. (2018). Saberes botánicos en el talar: utilización de plantas silvestres con fines medicinales y alimenticios en el parque costero del sur (partidos de Magdalena y Punta indio, Buenos Aires, República Argentina). Gaia Scientia 12: 56-80. https://doi.org/10.22478/ufpb.1981$1268.2018 \mathrm{v} 12 \mathrm{n} 1.32839$

GREIDER, T. \& L. GARKOVICH. (1994). Landscapes: The social construction of nature and the environment. Rural Sociology 59: 1-24. https://doi.org/10.1111/j.1549-0831.1994.tb00519.x

HILGERT, N., A. LAMBARÉ, N. D. VIGNALE, P. STAMPELLA \& M. L. POCHETTINO. (2014). Especies Naturalizadas o antropizadas? Apropiación local y la construcción de saberes sobre los frutales introducidos en época histórica en el norte de Argentina. Revista Biodiversidad Neotropical 4: 6987. https://doi.org/10.18636/bioneotropical.v4i2.118

HURRELL, J. A., P. C. STAMPELLA, M. B. DOUMECQ \& M. L. POCHETTINO. (2019). Ethnoecology in pluricultural contexts: Theoretical and methodological contributions. En ALBUQUERQUE, U., R. PAIVA DE LUCENA, L. FERNANDES DA CUNHA \& R. NÓBREGA (eds.), Methods and Techniques in Ethnobiology and Ethnoecology, pp. 163-186. Humana Press, Springer, New York.

https://doi.org/10.1007/978-1-4939-8919-5_12

IBODA. (2018). Instituto de Botánica Darwinion. Base de datos. Disponible: http://www.darwin. edu.ar/\#basededatos/floraargentina (Consulta: $18 / 11 / 2018)$.

INDEC. (2010). Censo Nacional de Población, Hogares y Viviendas, 2010. Disponible: http://www. censo2010.indec.gov.ar/ (Consulta: 12/12/2018).
INGOLD, T. (1993). The Temporality of the Landscape. World Archaeology 25: 152-174. https://doi.org/10.1080/00438243.1993.9980235

LAMBARÉ, D. A. (2015). Procesos locales de selección cultural en poblaciones frutales de la familia Rosaceae originarias del Viejo Mundo utilizados por comunidades rurales del noroeste argentino. Tesis Doctoral inédita. Facultad de Ciencias Naturales y Museo, UNLP.

MAFFI, L. (2001). Introduction. On the interdependence of biological and cultural diversity. En MAFFI, L. (ed.), On biocultural diversity. Linking language, knowledge, and the environment, pp. 1-50. Smithsonian Institution Press, Washington. https://doi.org/10.1093/jdh/14.4.253

MARTÍNEZ, G. J. \& J. MANZANO-GARCÍA. (2016). Estilos de percepción de la biodiversidad y su conservación en actores sociales de áreas protegidas de Córdoba. Revista del Museo de Antropología 9: 135-152. https://doi.org/10.31048/1852.4826.v9.n2.14169

PALEO, M. C., M. M. PÁEZ \& M. PÉREZ MERONI. (2002). Condiciones ambientales y ocupación humana durante el Holoceno tardío en el litoral fluvial bonaerense. En MAZZANTI, D., M. BERÓN \& F. OLIVA (eds.), Del Mar a los Salitrales, pp. 365376. Universidad Nacional de Mar del Plata, Mar del Plata.

PALEO, M. C. \& M. PÉREZ MERONI. (2004). Problemáticas vinculadas a las estrategias de subsistencia de la localidad arqueológica Barrio San Clemente. En GRADIN, C. J. \& F. OLIVA (eds.), El Área Pampeana. Su pasado arqueológico, pp. 311319. Laborde. Rosario, Universidad de Rosario y Municipalidad de Venado Tuerto.

PALEO, M. C. \& M. PÉREZ MERONI. (2007). Primeros resultados del sitio "Las Marías", Partido de Magdalena. Provincia de Buenos Aires. En OLIVA, F., N. DE GRANDIS \& J. RODRÍGUEZ (comps.), Arqueología Argentina en los inicios de un nuevo siglo I, pp. 275-286. Laborde, Rosario.

PALEO, M. C. \& M. PÉREZ MERONI. (2010). Del bosque de tala al Parque Costero del Sur. En BERÓN, M., L. LUNA, M. BONOMO, C. MONTALVO, C. ARANDA \& M. CARRERA (eds.), Mamül Mapu: Pasado y presente desde la arqueología pampeana 1, pp. 215-226. Libros Espinillos, Ayacucho.

PALEO, M. C., M. S. GARCÍA LERENA, P. STAMPELLA, M. B. DOUMECQ \& M. L. POCHETTINO. (2016). La construcción del paisaje del litoral rioplatense. Tomo 2: Las estancias y 
sus árboles. Editorial Universitaria de La Plata, Argentina. $72 \mathrm{pp}$.

PARODI, L. R. (1940). Distribución geográfica de los talares de la Provincia de Buenos Aires. Darwiniana 4: 33-56.

PÉREZ MERONI, M. \& M. C. PALEO. (1999). La utilización del espacio por grupos del litoral fluvial bonaerense. En LÓPEZ MAZZ, J. M. \& M. SANS (comps.), Arqueología y Bioantropología de las Tierras Bajas, pp. 165-172. Facultad de Humanidades y Ciencias de la Educación. Universidad de la República, Montevideo.

POCHETTINO, M. L., A. LAMBARÉ, P. STAMPELLA, M. B. DOUMECQ \& N. GHIANI ECHENIQUE. (2015). Especies arbóreas como texto en contextos pluriculturales. La conservación como pretexto. Acta de Reunión de Antropología del Mercosur. Disponible en: http://xiram.com.uy/actas-delcongreso/grupos-de-trabajo/ponencias-grupo-detrabajo-83 (Consulta: 15/03/2019).

RIAT, P. (2015). Puesta en valor de plantas subutilizadas: aporte a la conservación de los recursos naturales en Los Juríes (Santiago del Estero). Tesis Doctoral, Universidad Nacional de La Plata, Argentina.

RIAT, P., P. C. STAMPELLA \& M. L. POCHETTINO. (2018). Incidencia de la estrategia de uso múltiple en la autosubsistencia de dos comunidades campesinas de la Argentina. Gaia Scientia 12: 128-145. https://doi.org/10.22478/ufpb.1981$1268.2018 \mathrm{v} 12 \mathrm{n} 1.32837$

SALLERAS, L. (2013). Patrimonio cultural, territorio y turismo en la Quebrada de Humahuaca (Jujuy, Argentina): discusiones en torno al desarrollo. Actas del XXIX Congreso a las crisis y emergencias sociales en América Latina.

STAMPELLA, P. (2015). Historia local de naranja amarga (Citrus $\times$ aurantium L., Rutaceae) del Viejo Mundo asilvestrada en el corredor de las antiguas misiones jesuíticas de la provincia de Misiones (Argentina). Caracterización desde una perspectiva interdisciplinaria. Tesis Doctoral inédita. Facultad de Ciencias Naturales y Museo, UNLP.

STAMPELLA, P. (2018). Domesticación del paisaje en enclaves pluriculturales del sur de Misiones (Argentina): una aproximación a través de los cítricos. Boletín de la Sociedad Argentina de
Botánica 53: 135-150. https://doi.org/10.31055/1851.2372.v53.n1.19913

STAMPELLA, P., G. DELUCCHI \& M. L. POCHETTINO. (2013). Naturalización e identidad del "limón mandarina", Citrus $\times$ taitensis (Rutaceae, Aurantioideae) en la Argentina. Boletín de la Sociedad Argentina de Botánica 48: 161-169.

STAMPELLA, P., M. B. DOUMECQ, M. VOJKOVIC \& L. LABORDA. (2016). Valoración del cambio ambiental según los junqueros y leñateros en el sector sur de la región Rioplatense (Argentina). Bonplandia 25: 17-31. https://doi.org/10.30972/bon.2511268

TEJADA, M., E. CAROL \& E. KRUSE. (2011). Límites y potencialidades de las reservas de agua dulce en el humedal de la Bahía de Samborombón, Argentina. Revista de Geología Aplicada a la Ingeniería y al Ambiente 27: 57-61.

TORIBIO, A. \& C. SORUCO DE MADRAZO. (2005). Las instituciones académicas y su contribución al desarrollo sustentable. Estudios sobre Sociedad, Naturaleza y Desarrollo. Revista THEOMAI. Número especial. Disponible en: http://revistatheomai.unq.edu.ar/numespecial2005/art toribio_ numesp2005.htm (Consultada el 30/10/19).

TORRES ROBLES, S. S. (2009). Variación geográfica de la composición y riqueza de plantas vasculares en los talares bonaerenses y su relación con el clima, sustrato, estructura del paisaje y uso. Tesis Doctoral, Facultad de Ciencias Naturales y Museo, UNLP, Argentina.

TRENTINI, M. F. (2015). Pueblos indígenas y áreas protegidas: Procesos de construcción de identidades y territorialidades en el co-manejo del Parque Nacional Nahuel Huapi. Tesis Doctoral, Facultad de Filosofía y Letras, UBA, Argentina. https://doi.org/10.7440/res55.2016.02

TUXILL, J. \& G. P. NABHAN. (2001). Plantas, comunidades y áreas protegidas: una guía para el manejo in situ. Pueblos y Plantas vol. 3. Uruguay, Editorial Nordan-Comunidad.

UNESCO. (2017). Programa sobre el Hombre y la Biosfera. Disponible: http://www.unesco.org/ new/es/natural-sciences/environment/ecologicalsciences/man-and-biosphere-programme/ (Consulta: 15/03/2019). 